\title{
Pulsed photoelectric field emission from needle cathodes
}

\author{
C. Hernandez Garcia and C. A. Brau
}

Vanderbilt University, Department of Physics, Nashville, TN 37235, USA

Experiments have been carried out to measure the current emitted by tungsten needles with $1-\mu \mathrm{m}$ tip radius operated up to $50 \mathrm{kV}$. This corresponds to electric fields on the order of $10^{9}$ to $10^{10} \mathrm{~V} / \mathrm{m}$. The needles were illuminated with $10-\mathrm{ns}$ laser pulses at $532 \mathrm{~nm}, 355 \mathrm{~nm}$ and $266 \mathrm{~nm}$. The laser intensity was varied from $10^{10} \mathrm{~W} / \mathrm{m}^{2}$ to $10^{12} \mathrm{~W} / \mathrm{m}^{2}$, limited by damage to the needle tip. The observed quantum efficiency depends on the wavelength and the electric field, approaching unity at the highest electric fields when illuminated at $266 \mathrm{~nm}$. Peak currents up to $100 \mathrm{~mA}$ were observed in nanosecond pulses, corresponding to an estimated brightness of $10^{16} \mathrm{~A} / \mathrm{m}^{2}$-steradian. Since the current is controlled by the laser intensity, with only a weak voltage dependence, these cathodes can be used for infrared and ultraviolet tabletop free-electron lasers and other applications that demand short electron-beam pulses with high brightness.

\section{INTRODUCTION.}

The development of compact free-electron lasers has been paced by the search for better electron beams [1]. Presently available e-beams for FELs have a normalized brightness on the order of $10^{11} \mathrm{~A} / \mathrm{m}^{2}$-steradian, [2] compared with $10^{16} \mathrm{~A} / \mathrm{m}^{2}$-steradian for field-emission beams from needle cathodes [3]. An e-beam with a brightness of this order of magnitude could make possible the construction of tabletop devices such as the Smith-Purcell FEL [45] operating in the far-infrared or the Thomson FEL operating in the ultraviolet region [2. However, field emission is difficult to control and typically exhibits large fluctuations, both of which limit its usefulness. Photoelectric field emission is much more stable and easier to control.

Needle cathodes operated at high surface electric fields emit electrons by field emission. The emission mechanism involves tunneling through the barrier at the surface of the needle. Field emission is enhanced if the surface is irradiated with a laser that excites the electrons to levels above the Fermi level where the surface barrier is thinner, even if $\hbar \omega$ is less than the barrier height. Energy-resolved experiments by Venus and Lee using $\mathrm{cw}$ irradiation indicate a quantum efficiency on the order of $10^{-6}$ for emission to take place before the electrons relax to lower energy levels [6]. Other experiments indicate that most of the electrons relax to energy levels just above the Fermi level before they tunnel out [7]. The emission depends on the incident intensity in a nonlinear fashion that is explained by a competition between tunneling and relaxation.

In experiments using $248 \mathrm{~nm}, 337 \mathrm{~nm}$, and $350 \mathrm{~nm}$ laser pulses at intensities on the order of $10^{10}-10^{11} \mathrm{~W} / \mathrm{m}^{2}$, Ramian and Garate observe a weak dependence of the current on the surface electric field and quantum efficiencies on the order of unity at all wavelengths [8]. The effect is interpreted as single-photon photoemission in which the electron is excited to an energy level above the barrier, which is lowered by the Schottky 
effect. In similar experiments by Boussoukaya, et al., the tip is heated by the laser pulse and thermionic emission could account for the observed current [P].

In the present experiments tungsten needles are illuminated at intensities from $10^{10} \mathrm{~W} / \mathrm{m}^{2}$ to $10^{12} \mathrm{~W} / \mathrm{m}^{2}$ in 10 -nanosecond pulses at $532 \mathrm{~nm}, 355 \mathrm{~nm}$, and $266 \mathrm{~nm}$. The emission depends strongly on the wavelength. The quantum efficiency at $266 \mathrm{~nm}$ depends on the electric field but approaches unity at the highest fields.

\section{EXPERIMENTAL TECHNIQUES.}

The apparatus used in the present experiments is described elsewhere [7] Polycrystalline tungsten wire is etched to produce a needle with a tip radius of the order of $0.5-1.0 \mu \mathrm{m}$. The needle is heated to $2200 \mathrm{C}$ by electron bombardment to produce a smooth, clean surface. Contamination is minimized by maintaining the background pressure at $5 \times 10^{-11}$ Torr, and the needle is cleaned before every experimental run by heating it for a few minutes to $1800 \mathrm{C}$ with a $1-\mathrm{W} \mathrm{cw}$ argon-ion laser focused on one side of the tip. The current is measured at the anode with a response time around $3 \mathrm{~ns}$.

A Q-switched Nd:YAG laser is used to generate pulses about 10-ns long at the frequency doubled, tripled, and quadrupled wavelengths of the laser. By adjusting the distance between the tip and the focusing lens, the intensity can be varied from $10^{10}$ to $10^{12} \mathrm{~W} / \mathrm{m}^{2}$. At $532 \mathrm{~nm}$ the spot size at the focus is about $50 \mu \mathrm{m}$, at $355 \mathrm{~nm}$ it is about $100 \mu \mathrm{m}$, and at $266 \mathrm{~nm}$ about $50 \mu \mathrm{m}$. The instantaneous power is monitored with a response time of about 2 ns and a timing uncertainty around 2 ns.

\section{EXPERIMENTAL RESULTS.}

After the needle tip is smoothed by electron bombardment and cleaned with the cw laser, the ordinary field-emission current follows a strongly voltage-dependent straight line on a Fowler-Nordheim plot [7]. The tip radius determined from these measurements is within $10 \%$ of the value determined by electron microscope pictures.

When the cathode is illuminated by the pulsed laser the current is as shown in Figure 1. The spikes observed in the laser pulse are caused by temporal mode beating. In experiments at $532 \mathrm{~nm}$ no current is observed until the laser intensity reaches $8 \times 10^{11}$

$\mathrm{W} / \mathrm{m}^{2}$. This is near the known threshold for uv laser ablation of tungsten [10]. After ablation occurs the needles are heated to $2200 \mathrm{C}$ for a few hours to recover a smooth tip.

Similar ablation effects are observed at $355 \mathrm{~nm}$ and $266 \mathrm{~nm}$. However, the pulse current is linear in the laser intensity below the ablation threshold, as shown in Eigure 2. The measured quantum efficiency is the ratio of the total number of electrons in the current pulse and the total number of photons incident on the tip during the laser pulse. The quantum efficiency at $266 \mathrm{~nm}$ is about two orders of magnitude larger than that at $355 \mathrm{~nm}$, and increases with the surface electric field as shown in Figure 3

\section{INTERPRETATION}

The dependence of the quantum efficiency on the electric field at $266 \mathrm{~nm}$ can be interpreted by means of the simple model illustrated in Figure 4 , where the energy $E$ of the electrons is plotted as a function of the distance $z$ normal to the surface of the metal. If the electrons in the metal behave as a free electron gas, the density of electron states is 
$\rho(E) \propto E^{1 / 2}$. Photoemission is presumed to occur if the photon energy is enough to raise an electron to an energy level above the peak of the barrier at the surface of the needle. The Schottky effect lowers the barrier $V_{\max }$ below the work function $\phi$ by the amount $\left(q^{3} F / 4 \pi \varepsilon_{0}\right)^{1 / 2}$, where $q$ is the electron charge, $F$ the electric field, and $\varepsilon_{0}$ the permittivity of free space [H]. A photon of energy $\hbar \omega$ can excite electrons to levels above the barrier if the initial electron energy is in the energy band between $V_{\max }-\hbar \omega$ and the Fermi energy $E_{F}$, as shown in Figure 4 . If all electrons have equal probability of photoexcitation and all electrons excited above the barrier have unit probability for escape, the quantum efficiency is

$$
\eta(F)=\frac{N_{e}(F)}{N_{0}}=1-\left[1+\frac{\phi-\hbar \omega}{E_{F}}-\frac{1}{E_{F}} \sqrt{\frac{q^{3} F}{4 \pi \varepsilon_{0}}}\right]^{3 / 2} .
$$

This is shown by the curve $\mathrm{A}$ in Figure 3, and agrees reasonably well with the experimental data. Curve $\mathrm{B}$ is a $\sqrt{F}$ fit to the data.

This model does not, however, explain the quantum efficiency observed at 355 $\mathrm{nm}$. As discussed above, the quantum efficiency is much lower at $355 \mathrm{~nm}$ even when the photon energy exceeds the surface barrier. Nor does the model explain the ordinary quantum efficiency of tungsten observed in experiments conducted in the far ultraviolet at low surface electric fields where the Schottky effect is negligible.

\section{CONCLUSIONS}

The experimental quantum efficiency at $266 \mathrm{~nm}$ is observed to approach unity for the highest applied electric fields. A simple model for the quantum efficiency makes a good fit to the experimental data at $266 \mathrm{~nm}$. However, the model does not explain the data at $355 \mathrm{~nm}$. The largest current observed at $266 \mathrm{~nm}$ is about $100 \mathrm{~mA}$ from a $0.6-\mu \mathrm{m}$ tip. This corresponds to a current density $J=10^{11} \mathrm{~A} / \mathrm{m}^{2}$. If the electron temperature in the present experiments is on the order of $1 \mathrm{eV}$, the corresponding normalized brightness of the beam is on the order of $10^{16} \mathrm{~A} / \mathrm{m}^{2}$-steradian [12]. This is about four orders of magnitude larger than the brightness of conventional electron-beam sources. An electron beam with a brightness of this order is sufficient to develop tabletop free-electron lasers operating from the far infrared to the ultraviolet and soft $\mathrm{x}$-ray regions [2].

\section{ACKNOWLEDGEMENTS}

The authors gratefully appreciate helpful and illuminating discussions with Drs. Leonard Feldman, Richard Haglund, Sokrates Pantelides, and Thomas Weiler from Vanderbilt University, and Gerald Ramian from the University of California at Santa Barbara. Special thanks are due Drs. Marcus Mendenhall, William Gabella, and John Kozub from the Vanderbilt FEL for their help in the implementation of many aspects of the experimental apparatus and the data acquisition techniques. This work was supported by the Office of Naval Research. 


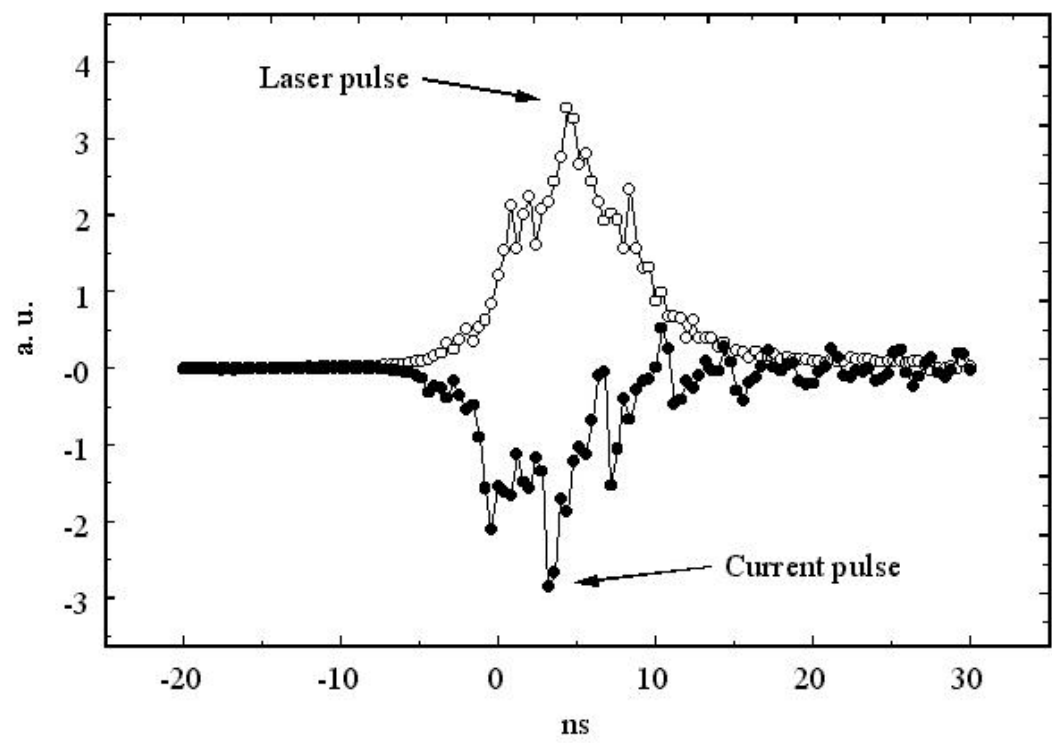

Figure 1 Typical laser pulse at $266 \mathrm{~nm}$ and current pulse (both traces in arbitrary units) versus time (ns). The maximum in the current pulse corresponds to $112 \mathrm{~mA}$.

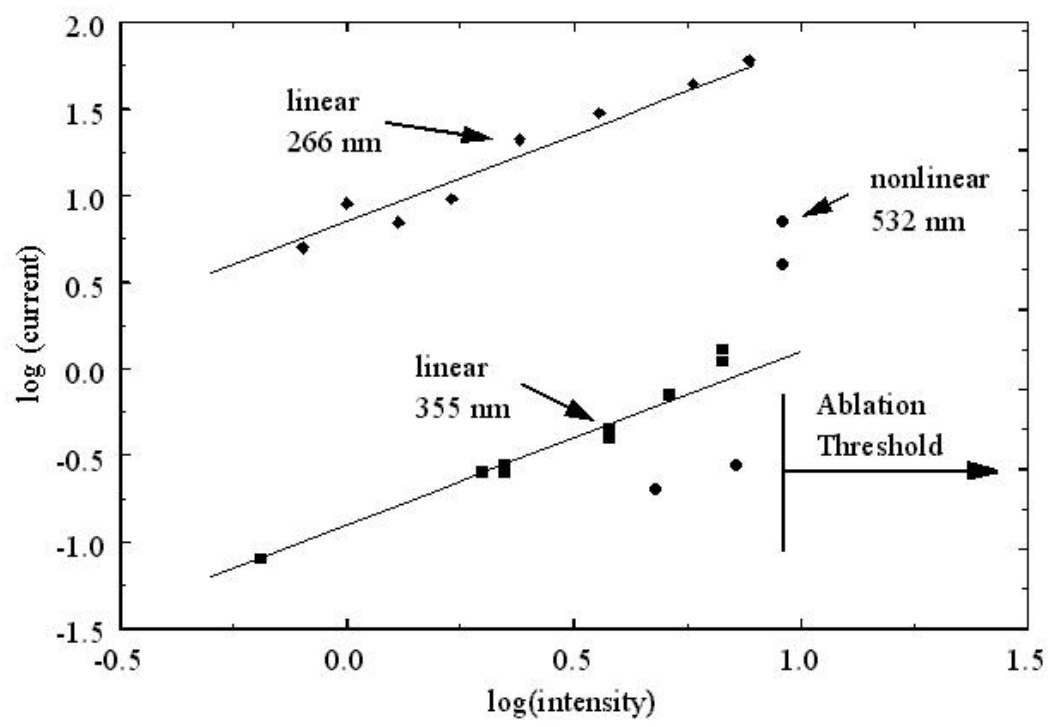

Figure 2 Double-logarithmic plot of the pulsed current $(\mathrm{mA})$ vs. laser intensity $\left(10^{11}\right.$ $\mathrm{W} / \mathrm{m}^{2}$ ). The surface electric field was $1.5 \times 10^{9} \mathrm{~V} / \mathrm{m}$ for $266 \mathrm{~nm}, 7.0 \times 10^{9} \mathrm{~V} / \mathrm{m}$ for $355 \mathrm{~nm}$ and $1.0 \times 10^{9} \mathrm{~V} / \mathrm{m}$ for $532 \mathrm{~nm}$. 


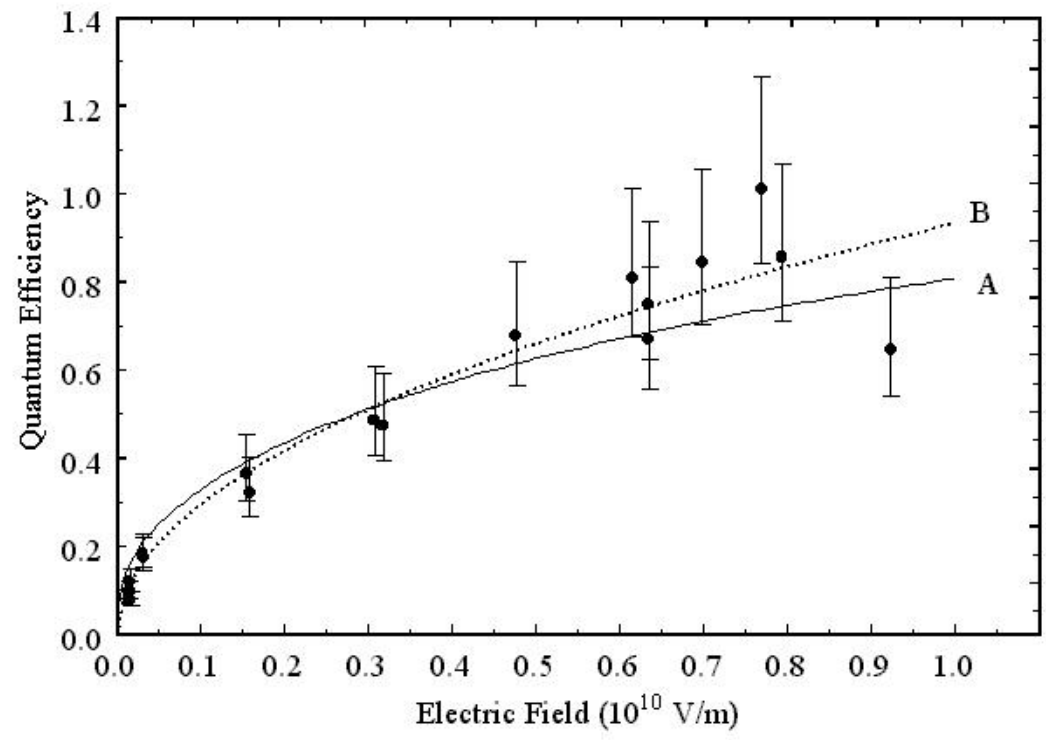

Figure 3 Quantum efficiency at $266 \mathrm{~nm}$. Curve A is computed from (1), Curve B is a $\sqrt{F}$ fit to the data, where $F$ is the surface electric field.

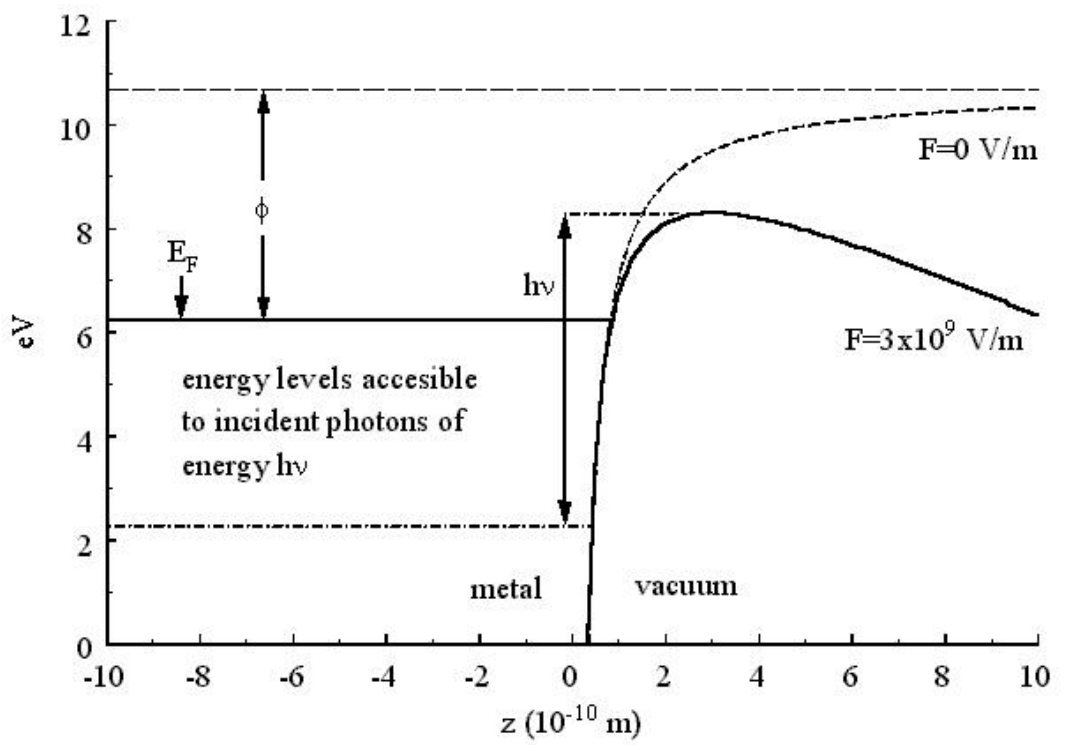

Figure 4 Electron energy $E$ vs. distance $z$ normal to the surface of the metal. $E_{F}$ is the Fermi energy and $\phi$ is the work function of the metal. The number of electrons that can be directly photoemitted increases when the electric field $F$ lowers the peak of the barrier (called the Schottky effect). 


\section{REFERENCES}

1 C. A. Brau, Free-Electron Lasers, (Academic Press, Boston, 1990), p. 277-286.

2 C. A. Brau, Nucl. Instr. And Meth. A 407, 1 (1998)

3 W. P. Dyke, W. W. Dolan, Advances in Electronics and Electron Physics, edited by L. Marton, Vol. VIII, Academic Press, New York (1956) 168.

4 J. M. N. Urata, Thesis, Dartmouth College, April 1997

5 J. E. Walsh, J. H. Brownell, J. C. Swartz, J. Urata, and M. F. Kimmitt, Nucl. Instr. And Meth A 407 (1999) 457.

6 D. Venus, M. J. G. Lee, Surf. Sci. 116, 359 (1982).

7 C. Hernandez Garcia, and C. A. Brau, Nucl. Instr. And Meth. A 429, 257 (1999).

8 G. Ramian, E. Garate (private communication).

9 M. Boussoukaya, H. Bergeret, R. Chehab, B. Leblond, and J. Le Duff, Nucl. Instr. And Meth. A 279, 405 (1989).

10 C. Beleznai, D. Vouagner, J.P Girardeau-Montaut, Appl. Surf. Sci. 127, 88 (1998).

11 A. Modinos, Field, Thermionic, and Secondary Electron Emission Spectroscopy (Plenum Press, New York, 1984), p. 8.

12 J. D. Lawson, The Physics of Charged-Particles Beams (Clarendon Press, Oxford, 1988), p. 160. 